\title{
CIRUGÍA AMBULATORIA: SELECCIÓN DE PACIENTES Y PROCEDIMIENTOS QUIRÚRGICOS*
}

\author{
Dr. Claudio Nazar J. ${ }^{1}$, Int. Maximiliano Zamora H. ${ }^{2}$, Dr. Alejandro González A. ${ }^{1}$ \\ División de Anestesiología. \\ 2 Interno de Medicina. \\ Escuela de Medicina, Facultad de Medicina, Pontificia Universidad Católica de Chile. \\ Santiago, Chile.
}

\begin{abstract}
\section{Ambulatory surgery: patients and surgeries selection}

Outpatient surgery is being performed with increasing frequency due to its significant benefits, including lower costs in health care, and lower incidence of complications and mortality, requiring an appropriate selection of patients and surgeries to be performed in this setting. To select patients and surgeries to be operated on an ambulatory basis, it is relevant an adequate preoperative evaluation. Regarding the risks of patient, it is important the comorbidities and the American Society of Anesthesiologists classification. Risks associated with the type of surgery are divided according to their cardiovascular risk and duration of the procedure. Both will define those suitable for outpatient surgery. Serious complications and associated mortality are infrequents nowadays, therefore it is necessary to take into account other indicators, such as unanticipated hospital admission, hospital readmission and prolonged postoperative stay. There are some patients that require more specific preoperative evaluation, such as the elderly, obese, among others.
\end{abstract}

Key words: Ambulatory surgical procedures, ambulatory anesthesia, patient selection, surgery selection, perioperative care, risk assessment, risk factors.

\section{Resumen}

La cirugía ambulatoria se realiza cada vez con mayor frecuencia debido a sus importantes beneficios, como menores costos y menor morbimortalidad, y requiere una cuidadosa selección de pacientes y procedimientos quirúrgicos a realizar. Para seleccionar qué pacientes se pueden operar en forma ambulatoria es necesaria una adecuada evaluación preoperatoria quirúrgica y anestésica. Respecto a los riesgos asociados al paciente, destacan sus comorbilidades y clasificación American Society of Anesthesiologists. Los riesgos asociados a la cirugía se dividen según su riesgo cardiovascular y duración del procedimiento. Entre los riesgos del paciente y la cirugía se pueden definir cuáles son aptos para una cirugía ambulatoria. Las complicaciones graves y mortalidad asociadas son muy infrecuentes hoy en día, por lo que se hace necesario tener en cuenta

*Recibido el 21 de agosto de 2014 y aceptado para publicación el 23 de septiembre de 2014.

Los autores no refieren conflictos de interés.

Correspondencia: Dr. Alejandro González A.

gonzalea@med.puc.cl 
otro tipo de indicadores, como la hospitalización no programada, hospitalización post alta y retardo del alta hospitalaria. Existen ciertos pacientes que requieren una evaluación más específica de la indicación de cirugía ambulatoria, como los ancianos y obesos, entre otros.

Palabras clave: Cirugía ambulatoria, anestesia ambulatoria, selección de pacientes, selección de cirugías, cuidados perioperatorios, evaluación de riesgos, factores de riesgo.

\section{Introducción}

La cirugía ambulatoria (CA) definida como aquellos procedimientos quirúrgicos electivos, en que la admisión, la cirugía y el alta del paciente ocurren el mismo día, requiere una cuidadosa selección de pacientes y cirugías ${ }^{1}$.

La CA ha presentado un aumento significativo durante las últimas décadas a nivel mundial. En países desarrollados los procedimientos quirúrgicos ambulatorios representan sobre el $60 \%$ del total de cirugías electivas $^{2}$. Este incremento se explica principalmente por los avances en las técnicas quirúrgicas y anestésicas, lo que también ha permitido que un número creciente de pacientes y/o procedimientos más complejos sean hoy considerados para $\mathrm{CA}^{2}$.

En Chile también ha habido un alza en la CA, existiendo distintos programas de CA con resultados satisfactorios y muy bajas complicaciones ${ }^{3,4}$. Hasta donde sabemos, no hay estadísticas nacionales que indiquen el porcentaje de cirugías electivas realizadas en modalidad ambulatoria, sin embargo, creemos que es bastante menor que el reportado en países desarrollados. En nuestro centro, estadísticas no publicadas muestran que aproximadamente un $15 \%$ de los procedimientos quirúrgicos electivos se realizan de forma ambulatoria.

Los beneficios de la CA, que hacen de esta una excelente alternativa, son su menor costo económico para los pacientes y los centros asistenciales, recuperación más rápida del estado fisiológico preoperatorio del paciente y reintegración más precoz a sus actividades habituales, menor morbimortalidad y mayor satisfacción usuaria ${ }^{5}$. Los menores costos de la CA en ningún modo deben alterar los estándares de seguridad y calidad de la atención de los pacientes.

A nuestro entender, la CA se apoya en tres pilares fundamentales: el lugar donde se realiza, esto es la implementación y el tipo de centro de CA; la adecuada y cuidadosa selección de los pacientes y cirugías a realizar de forma ambulatoria, y el sistema de control y apoyo posterior al alta. En el presente artículo revisaremos qué factores se deben considerar para la selección de pacientes y de procedimientos quirúrgicos para $\mathrm{CA}$.

La evaluación preoperatoria es la que permite identificar qué pacientes y qué cirugías son adecuados para $\mathrm{CA}$, como también determinar y reducir los factores de riesgo de complicaciones. Consideramos que en este proceso y en la selección caso a caso ayuda resolver las siguientes interrogantes: ¿cuál es la extensión de la enfermedad y cuál es el estado funcional del paciente?; ¿está el tratamiento médico optimizado?; ¿en qué grado la cirugía altera o descompensa la enfermedad de base?; ¿existe riesgo de dar de alta el mismo día o tendrá el paciente un manejo más óptimo hospitalizado?

\section{¿Qué pacientes operar?}

Existen varias formas de realizar una evaluación preoperatoria, sin embargo, es el cirujano el primero en evaluar al paciente y en decidir la cirugía. En este sentido, es importante que él esté familiarizado con los requerimientos de la evaluación y que exista una fluida comunicación entre cirujano y anestesista, de modo que el proceso de evaluación, selección y preparación preoperatoria sea eficiente ${ }^{6}$. La evaluación y optimización de la condición preoperatoria basal del paciente deben ser acuciosas y con la debida anticipación, de manera de evitar postergaciones o suspensiones de última hora ${ }^{7}$.

Existen varios criterios descritos en la literatura que permiten una adecuada selección de pacientes ${ }^{8,9}$, considerando que el riesgo está determinado principalmente por las comorbilidades asociadas. El más frecuentemente utilizado es la clasificación de estado físico de la American Society of Anesthesiology o $\mathrm{ASA}^{10}$ (Tabla 1), que refleja la directa asociación entre las comorbilidades del paciente y su riesgo de mortalidad perioperatoria.

En líneas generales, los pacientes ASA I y II son candidatos para realizarse procedimientos quirúrgicos en forma ambulatoria ${ }^{6}$. Los pacientes portadores de diversas patologías pueden ser candidatos a una CA siempre y cuando tengan un manejo y control adecuado de su(s) enfermedad(es) de base, o bien, cuando es posible optimizar su condición basal y tratamiento con debida antelación antes de un procedimiento quirúrgico. Los pacientes ASA III podrían ser sometidos a una CA de bajo riesgo cardiovascular (riesgo menor al 1\% de morbimortalidad cardiovascular) ${ }^{11}$ solamente si tienen un control y tratamiento óptimos de su(s) comorbilidad(es), ya que en caso contrario deberían quedar hospitalizados debido a que tiene un mayor riesgo de complicaciones postoperatorias ${ }^{6}$. 
Tabla 1. Clasificación de estado físico de la American Society of Anesthesiologists (ASA) ${ }^{10}$

\begin{tabular}{|clc|}
\hline Clasificación ASA & Descripción & Mortalidad perioperatoria \\
\hline I & Paciente sano, sin comorbilidad & $0-0,3 \%$ \\
II & Enfermedad sistémica leve a moderada, sin limitación funcional & $0,3-1,4 \%$ \\
III & Enfermedad sistémica moderada a severa, con limitación funcional & $1,8-5,4 \%$ \\
IV & Enfermedad sistémica severa, con riesgo vital constante & $7,8-25,9 \%$ \\
V & Paciente moribundo, con pocas probabilidades de sobrevivir las próxi- & $9,4-57,8 \%$ \\
& mas 24 h, con o sin cirugía & $100 \%$ \\
\hline
\end{tabular}

Tabla 2. Tipos de cirugía agrupados según riesgo cardiovascular ${ }^{11}$

\begin{tabular}{|c|c|c|}
\hline Riesgo bajo (menor a 1\%) & Riesgo intermedio (1-5\%) & Riesgo alto (sobre 5\%) \\
\hline Procedimientos endoscópicos & Endarterectomía carotidea & $\begin{array}{l}\text { Cirugías de emergencia mayores, sobre- } \\
\text { todo en adultos mayores }\end{array}$ \\
\hline $\begin{array}{l}\text { Procedimientos en órganos superficiales } \\
\text { (por ejemplo herniorrafia) }\end{array}$ & Cirugía de cabeza y cuello & Cirugías vasculares: aórticas y otras \\
\hline Cirugía mamaria & Cirugía intraperitoneal e intratorácica & Cirugía vascular periférica \\
\hline \multirow[t]{2}{*}{ Cirugía de cataratas } & Cirugía ortopédica & $\begin{array}{l}\text { Cirugías largas }(>3 \mathrm{~h}) \text { con gran recam- } \\
\text { bio de fluidos y/o pérdidas sanguíneas }\end{array}$ \\
\hline & Cirugía prostática & \\
\hline
\end{tabular}

En cuanto a los exámenes preoperatorios para este tipo de pacientes, existen estudios que confirman que en CA de bajo riesgo no es necesario solicitar exámenes de rutina, a menos que alguna patología del paciente lo amerite. Estos exámenes están alterados en menos de un 1\% de los casos y, en caso de estar alterados, no se asocian con mayores complicaciones en el período postoperatorio ${ }^{12}$. Sin embargo, cuando los exámenes preoperatorios están bien indicados, podrían reducir el riesgo perioperatorio al permitir optimizar el estado basal del paciente $^{6,13}$. Por lo tanto, para solicitar exámenes preoperatorios se deben utilizar los mismos criterios que aquellos empleados para pacientes quirúrgicos hospitalizados $^{13}$.

\section{¿Qué cirugías realizar?}

El riesgo asociado al tipo de cirugía también debe ser considerado al momento de seleccionar a un paciente para CA. La American Heart Association (AHA) y American College of Cardiology (ACC), identifican tres tipos de cirugía según su riesgo de complicaciones cardiovasculares perioperatorias
(Tabla 2$)^{11}$ : riesgo bajo $(<1 \%)$, intermedio $(1-5 \%)$ $\mathrm{y}$ alto $(>5 \%)$. En este último grupo se recomienda hospitalizar al paciente rutinariamente debido a las razones anteriormente explicadas. Por lo mismo, toda cirugía de urgencia o emergencia no se puede realizar en forma ambulatoria. Otros requisitos para la selección de cirugías son que éstas tengan un mínimo de alteraciones fisiológicas postoperatorias, baja probabilidad de complicaciones, mínimo sangrado, dolor postoperatorio controlable con analgesia habitual y sin necesidad de uso de drogas endovenosas.

En relación a la duración del procedimiento quirúrgico, se ha demostrado que las cirugías ambulatorias de más de dos horas de extensión se relacionan a un riesgo aumentado de admisión hospitalaria no programada $^{14}$. Sin embargo, una duración estimada mayor a 2 h en una cirugía electiva no es una contraindicación de realizarla ambulatoriamente.

Se deben evaluar los riesgos del paciente y procedimiento quirúrgico por separado y en conjunto, debiendo ser discutidos por cirujano y anestesiólogo tratantes para, conjuntamente, evaluar la posibilidad de realizar el procedimiento quirúrgico de forma ambulatoria. En la Tabla $3^{6}$, se muestra la relación 
Tabla 3. Recomendaciones para realizar cirugías en forma ambulatoria (adaptado de Gupta $\mathbf{A}^{6}$ )

\begin{tabular}{|lccc|}
\hline Cirugía & Riesgo bajo & Paciente & Riesgo intermedio \\
Riesgo bajo & Sí & Probablemente sí* & No \\
Riesgo intermedio & Probablemente sí* & Probablemente no* & No \\
Riesgo alto & No & No & No \\
\hline
\end{tabular}

Riesgo de la cirugía, ver Tabla 2. Riesgo del paciente: riesgo bajo, ASA I-II; riesgo intermedio, ASA III; riesgo alto, ASA IV-V.

*Depende de la experiencia del servicio quirúrgico y de la disponibilidad de recursos.

entre el grado de riesgo de paciente y la cirugía, y la recomendación en cuanto a la indicación o no de una $\mathrm{CA}$. De esta se desprende que un paciente con clasificación I o II puede ser sometido hasta cirugías de riesgo cardiovascular intermedio en forma ambulatoria, así como un ASA III a cirugías de riesgo bajo, si se encuentra óptimamente compensado.

\section{Riesgos para el paciente y evaluación de resultados}

Actualmente las complicaciones más graves (falla respiratoria, infarto agudo al miocardio, trombo embolismo pulmonar) y mortalidad en CA son muy poco frecuentes. Se reportan mortalidades tan bajas como 1 en 11.273 pacientes, e índices de efectos adversos (morbilidad mayor) de $0,08 \%{ }^{15}$.

La mortalidad relacionada exclusivamente a la anestesia es muy infrecuente ${ }^{16}$, especialmente en el contexto ambulatorio. Hoefflin et al. ${ }^{17}$, reportaron más de 23.000 procedimientos quirúrgicos ambulatorios bajo anestesia general, sin complicaciones anestésicas mayores (Tabla 4) ${ }^{17}$ ni mortalidad. Por otro lado, Bitar et al. ${ }^{18}$, reportaron 4.778 pacientes sometidos a cirugía plástica de forma ambulatoria, bajo sedación endovenosa, también sin complicaciones anestésicas mayores ni mortalidad. En Chile, Correa ${ }^{19}$ reportó más de 20 años de experiencia en anestesia para procedimientos quirúrgicos ambulatorios, con 8.530 pacientes sometidos a sedación endovenosa y 220 a anestesia general, sin complicaciones anestésicas mayores ni mortalidad. En las tres series descritas la principal complicación fueron las náuseas y vómitos postoperatorias (NVPO) con una incidencia entre 0,2 y $25 \%$; aunque otras series reportan hasta un $35 \%$ de incidencia ${ }^{20,21}$.

Es por esto que se utilizan otros indicadores, que son más fidedignos, para evaluar los resultados de la CA, como son el retardo del alta, la hospitalización no programada y la hospitalización post alta. Entre los factores que se han asociado con retardo del alta hospitalaria destacan las NVPO y el dolor postope- ratorio. Las NVPO también son la primera causa de hospitalización no programada en $\mathrm{CA}^{22-24}$, además producen insatisfacción usuaria y reclamos de los pacientes en cirugía general ${ }^{25,26}$, siendo un efecto adverso frecuente e importante de prevenir ${ }^{27}$.

Los factores que se han asociado con riesgo elevado de hospitalización post alta de CA son la edad avanzada ( $\geq 65$ años), enfermedad vascular periférica, cardíaca, cerebrovascular, oncológica y paciente VIH positivo ${ }^{14}$. La anestesia general también se ha relacionado con riesgo aumentado de readmisión hospitalaria ${ }^{14}$.

\section{Pacientes y patologías específicas}

Además de lo expuesto anteriormente, existen situaciones clínicas de especial interés en cuanto a la selección de pacientes para este tipo de cirugías, las que se exponen a continuación:

\section{Tabla 4. Complicaciones anestésicas mayores ${ }^{17}$}

Accidente vascular encefálico
Paro cardiorrespiratorio
Infarto agudo al miocardio
Tromboembolismo pulmonar
Aspiración de contenido gástrico
Emergencia hipertensiva arterial
Falla hepática aguda
Falla renal aguda
Anafilaxia (fármacos, látex)
Hipertermia maligna
Convulsiones
Hospitalización secundaria a cualquier complicación
Requerimiento de ventilación mecánica no programada




\section{Adultos mayores}

En el contexto de un envejecimiento poblacional global, cada vez se presenta un número creciente de pacientes adultos mayores (sobre 65 años) para intervenirse quirúrgicamente de forma ambulatoria. Actualmente en nuestro país, este grupo de pacientes representa aproximadamente el $10 \%$ de la población ${ }^{28}$. La edad avanzada por sí misma no representa necesariamente una contraindicación para este tipo de procedimientos, debiendo considerarse también las comorbilidades del paciente, su estado general funcional actual y sus reservas fisiológicas ${ }^{29}$. Sin embargo, en pacientes mayores de 85 años existe un riesgo estadísticamente significativo aumentado de mortalidad perioperatoria luego de una CA, por lo que se recomienda su admisión hospitalaria luego de la cirugía ${ }^{6,30}$.

Si bien la edad avanzada y el antecedente de hipertensión arterial en este tipo de pacientes predisponen a mayores cambios hemodinámicos intraoperatorios, en general éstos no se asocian con grandes efectos adversos ni morbilidad postoperatoria. Así, un paciente de 70 años e hipertenso arterial crónico podría ser sometido a un procedimiento ambulatorio sin mayores inconvenientes 8 .

\section{Síndrome de apnea e hipopnea obstructiva del sueño}

Los pacientes portadores de síndrome de apnea e hipopnea obstructiva del sueño (SAHOS) se caracterizan por presentar obstrucción parcial o completa de la vía aérea superior durante el sueño, lo que produce hipoxia, hipercapnia y microdespertares con el fin de mantener la vía aérea permeable ${ }^{31}$. Varias complicaciones postoperatorias se han asociado con esta enfermedad, destacando hipoxemia, arritmias cardíacas, daño isquémico miocárdico, admisión no anticipada a la unidad de cuidados intensivos y muerte súbita ${ }^{32,33}$. Su prevalencia es alta, pudiendo estar presente hasta en un $4 \%$ de los hombres y $2 \%$ de las mujeres que son sometidos a algún tipo de procedimiento quirúrgico ${ }^{8}$; sin embargo, más de un $75 \%$ de los pacientes con alto riesgo de presentar SAHOS no tienen diagnóstico previo a su cirugía ${ }^{34}$.

Existen cuestionarios para identificar los pacientes susceptibles de presentar esta patología en el período preoperatorio, que se relacionan con factores de riesgo conocidos de ocurrencia de la enfermedad, siendo la obesidad y la edad avanzada los de mayor importancia ${ }^{35}$. Estos cuestionarios también permiten determinar la severidad del SAHOS ${ }^{36-39}$.

En 2012, Joshi et al., publicaron una serie de recomendaciones respecto de los pacientes con
SAHOS sometidos a CA. Si un paciente no tiene diagnóstico previo de esta enfermedad, pero presenta una probabilidad alta de tenerla, se recomienda realizar la cirugía ambulatoriamente, sin necesidad de mayor estudio, siempre y cuando no exista otra contraindicación. Los pacientes con diagnóstico previo de SAHOS son compatibles con la mayor parte de las cirugías ambulatorias, con la condición de tener todas sus comorbilidades compensadas y manteniendo el uso del dispositivo CPAP (continous positive airway pressure) en el período postoperatorio. Idealmente, en estos pacientes se debe evitar el uso de opioides sistémicos por el mayor riesgo de depresión respiratoria y obstrucción de la vía aérea alta por excesiva sedación ${ }^{38}$.

\section{Pacientes obesos}

La obesidad, definida por un índice de masa corporal (IMC) mayor o igual a 30, es una entidad extremadamente frecuente en nuestro país, alcanzando hasta un $25 \%$ de la población general, según la encuesta nacional de salud ${ }^{40}$, por lo que muy probablemente enfrentemos este tipo de pacientes en procedimientos ambulatorios. En general, los pacientes obesos pueden ser sometidos a una CA ya que un IMC $\geq 30$ no se ha identificado como factor de riesgo significativo de complicaciones cardiovasculares perioperatorias. Sin embargo, en los pacientes con IMC $>50$ existe un riesgo mayor de efectos adversos respiratorios intraoperatorios, como dificultad en la ventilación y generación de atelectasias, además de episodios de hipoxia y broncoespasmo postoperatorios, por lo que se recomienda su admisión hospitalaria en centros especializados en caso de realizarse una cirugía?

\section{Alergia al látex}

Los pacientes con alergia al látex pueden ser sometidos a CA. En ellos se recomienda utilizar un pabellón libre de látex, realizar la cirugía a primera hora de la mañana, preparar la unidad de recuperación y pre alta también libre de látex y realizar un adecuado control posterior al alta.

\section{Hipertermia maligna}

Los pacientes con historia personal y/o familiar de hipertermia maligna, o con antecedentes de miopatías asociadas a esta patología, pueden someterse a procedimientos ambulatorios, siempre y cuando el equipo quirúrgico haya tomado todas las medidas 
necesarias para no gatillar una crisis de hipertermia maligna, no utilizando gases anestésicos halogenados ni succinilcolina. Se recomienda realizar la cirugía a primera hora de la mañana, controlar la temperatura corporal en todo el período postoperatorio $\mathrm{y}$, luego, en su domicilio durante las primeras $24 \mathrm{~h}^{41}$.

\section{Conclusiones}

En resumen, la CA es cada vez más frecuente debido, entre otros beneficios, a sus menores costos, menor morbimortalidad y mayor satisfacción usuaria, y requiere de una cuidadosa selección de pacientes y de cirugías.

La evaluación preoperatoria permite realizar esta selección, teniendo en cuenta los riesgos asociados al paciente, principalmente sus comorbilidades y clasificación ASA, como también los riesgos asociados a la cirugía, especialmente el riesgo cardiovascular relacionado al procedimiento.

La morbilidad mayor y mortalidad son muy infrecuentes en CA, por lo que utiliza otro tipo de indicadores de resultados, como la hospitalización no programada, la hospitalización post alta, el retardo del alta hospitalaria y la satisfacción usuaria.

\section{Referencias}

1. De Lathouwer C, Poullier JP. How much ambulatory surgery in the World in 1996-1997 and trends? Ambulatory surgery. 2000;8:191-210.

2. Cullen KA, Hall MJ, Golosinskiy A. Ambulatory surgery in the United States, 2006. National health statistics reports. 2009;11:1-25.

3. Brunetto B, Castilla A, Sacaluga F. La adenoamigdalectomía como un procedimiento ambulatorio. Rev Otorrinolaringol Cir Cabeza Cuello 1992;52:167-71.

4. Patillo J, Kusanovic R, Salas P, Reyes J, GarcíaHuidobro I, Sanhueza M, et al. Colecistectomía laparoscópica ambulatoria: Una experiencia factible en un hospital público chileno. Rev Med Chile 2004;132:429-36.

5. Shnaider I, Chung F. Outcomes in day surgery. Curr Opin Anaesthesiol. [Review]. 2006;19:622-9.

6. Gupta A. Preoperative screening and risk assessment in the ambulatory surgery patient. Curr Opin Anaesthesiol. [Review]. 2009;22:705-11.

7. Holt NF, Silverman DG, Prasad R, Dziura J, Ruskin KJ. Preanesthesia clinics, information management, and operating room delays: results of a survey of practicing anesthesiologists. Anesth Analg. 2007;104:615-8.

8. Bryson GL, Chung F, Finegan BA, Friedman Z, Miller $\mathrm{DR}$, van Vlymen J, et al. Patient selection in ambulatory anesthesia-an evidence-based review: part I.
Canadian journal of anaesthesia $=$ Journal canadien d'anesthesie. [Research Support, Non-U.S. Gov't Review]. 2004;51:768-81.

9. Bryson GL, Chung F, Cox RG, Crowe MJ, Fuller $\mathrm{J}$, Henderson $\mathrm{C}$, et al. Patient selection in ambulatory anesthesia-an evidence-based review: part II. Canadian journal of anaesthesia $=$ Journal canadien d'anesthesie. [Research Support, Non-U.S. Gov't Review]. 2004;51:782-94.

10. Wolters U, Wolf T, Stutzer H, Schroder T. ASA classification and perioperative variables as predictors of postoperative outcome. Br J Anaesth. [Clinical Trial Duplicate Publication]. 1996;77:217-22.

11. American College of Cardiology/American Heart Association Task Force on Practice G, American Society of E, American Society of Nuclear C, Heart Rhythm S, Society of Cardiovascular A, Society for Cardiovascular A, et al. ACC/AHA 2007 guidelines on perioperative cardiovascular evaluation and care for noncardiac surgery: executive summary: a report of the American College of Cardiology/American Heart Association Task Force on Practice Guidelines (Writing Committee to Revise the 2002 Guidelines on Perioperative Cardiovascular Evaluation for Noncardiac Surgery). Anesth Analg. [Practice Guideline]. 2008;106:685-712.

12. Benarroch-Gampel J, Sheffield KM, Duncan CB, Brown KM, Han Y, Townsend CM, Jr., et al. Preoperative laboratory testing in patients undergoing elective, lowrisk ambulatory surgery. Annals of surgery. [Evaluation Studies Research Support, N.I.H., Extramural Research Support, Non-U.S. Gov't]. 2012;256:518-28.

13. Nazar JC, Bastidas EJ, Lema FG. Exámenes preoperatorios de rutina en cirugía electiva: ¿cuál es la evidencia? Rev Ch Cir. 2014;66:188-93.

14. Fleisher LA, Pasternak LR, Lyles A. A novel index of elevated risk of inpatient hospital admission immediately following outpatient surgery. Archives of Surgery. [Multicenter Study Research Support, Non-U.S. Gov't]. 2007;142:263-8.

15. Warner MA, Shields SE, Chute CG. Major morbidity and mortality within 1 month of ambulatory surgery and anesthesia. JAMA. [Research Support, Non-U.S. Gov't]. 1993;270:1437-41.

16. Gupta A. Strategies for outpatient anaesthesia. Best Pract Res Clin Anaesthesiol. [Review]. 2004;18:675-92.

17. Hoefflin SM, Bornstein JB, Gordon M. General anesthesia in an office-based plastic surgical facility: a report on more than 23,000 consecutive office-based procedures under general anesthesia with no significant anesthetic complications. Plast Reconstr Surg. 2001;107:243-51; discussion 52-7.

18. Bitar G, Mullis W, Jacobs W, Matthews D, Beasley M, Smith K, et al. Safety and efficacy of office-based surgery with monitored anesthesia care/sedation in 4778 consecutive plastic surgery procedures. Plast Reconstr Surg. 2003;111:150-6; discussion 7-8. 
19. Correa O. Anestesia en la consulta. Una experiencia personal. Revista Chilena de Anestesia. 2005;34:159.

20. Carroll NV, Miederhoff P, Cox FM, Hirsch JD. Postoperative nausea and vomiting after discharge from outpatient surgery centers. Anesth Analg. [Research Support, Non-U.S. Gov't]. 1995;80:903-9.

21. Apfel CC, Philip BK, Cakmakkaya OS, Shilling A, Shi YY, Leslie JB, et al. Who is at risk for postdischarge nausea and vomiting after ambulatory surgery? Anesthesiology. [Research Support, Non-U.S. Gov't]. 2012;117:475-86.

22. Gold BS, Kitz DS, Lecky JH, Neuhaus JM. Unanticipated admission to the hospital following ambulatory surgery. JAMA. 1989;262:3008-10.

23. Fortier J, Chung F, Su J. Unanticipated admission after ambulatory surgery-a prospective study. Canadian journal of anaesthesia $=$ Journal canadien d'anesthesie. [Clinical Trial]. 1998;45:612-9.

24. Gundzik K. Nausea and vomiting in the ambulatory surgical setting. Orthopaedic nursing/National Association of Orthopaedic Nurses. [Review]. 2008;27:182-8.

25. Gan TJ. Postoperative nausea and vomiting-can it be eliminated? JAMA. [Review]. 2002;287:1233-6.

26. Gan T, Sloan F, Dear Gde L, El-Moalem HE, Lubarsky DA. How much are patients willing to pay to avoid postoperative nausea and vomiting? Anesth Analg. [Research Support, Non-U.S. Gov't]. 2001;92:393-400.

27. Macario A, Weinger M, Truong $P$, Lee M. Which clinical anesthesia outcomes are both common and important to avoid? The perspective of a panel of expert anesthesiologists. Anesth Analg. [Research Support, Non-U.S. Gov't]. 1999;88:1085-91.

28. Enfoque estadístico-Adulto Mayor, Boletín informativo del Instituto Nacional de Estadísticas, Agosto 2005.

29. Morgan GE, Mikhail MS, Murray MJ. Clinical anesthesiology, Chapter 44: Ambulatory, Nonoperating Room, $\&$ Office-Based Anesthesia. 5th ed. New York: Lange Medical Books/McGraw Hill, Medical Pub. Division; 2013.

30. Bettelli G. High risk patients in day surgery. Minerva Anestesiol. [Review]. 2009;75:259-68.

31. Adesanya AO, Lee W, Greilich NB, Joshi GP. Perioperative management of obstructive sleep apnea. Chest. [Review]. 2010;138:1489-98.

32. Liao P, Yegneswaran B, Vairavanathan S, Zilberman P,
Chung F. Postoperative complications in patients with obstructive sleep apnea: a retrospective matched cohort study. Canadian journal of anaesthesia = Journal canadien d'anesthesie. [Research Support, Non-U.S. Gov't]. 2009;56:819-28.

33. Moos DD, Prasch M, Cantral DE, Huls B, Cuddeford JD. Are patients with obstructive sleep apnea syndrome appropriate candidates for the ambulatory surgical center? AANA journal. [Review]. 2005;73:197-205.

34. Ankichetty S, Chung F. Considerations for patients with obstructive sleep apnea undergoing ambulatory surgery. Curr Opin Anaesthesiol. [Research Support, Non-U.S. Gov't Review]. 2011;24:605-11.

35. Young T, Peppard PE, Taheri S. Excess weight and sleep-disordered breathing. Journal of applied physiology. 2005;99:1592-9.

36. Chung F, Yegneswaran B, Liao P, Chung SA, Vairavanathan S, Islam S, et al. Validation of the Berlin questionnaire and American Society of Anesthesiologists checklist as screening tools for obstructive sleep apnea in surgical patients. Anesthesiology. [Research Support, Non-U.S. Gov't]. 2008;108:822-30.

37. Chung F, Yegneswaran B, Liao P, Chung SA, Vairavanathan S, Islam S, et al. STOP questionnaire: a tool to screen patients for obstructive sleep apnea. Anesthesiology. [Research Support, Non-U.S. Gov't]. 2008;108:812-21.

38. Joshi GP, Ankichetty SP, Gan TJ, Chung F. Society for Ambulatory Anesthesia consensus statement on preoperative selection of adult patients with obstructive sleep apnea scheduled for ambulatory surgery. Anesth Analg. [Review]. 2012;115:1060-8.

39. Chung F, Subramanyam R, Liao P, Sasaki E, Shapiro C, Sun Y. High STOP-Bang score indicates a high probability of obstructive sleep apnoea. Br J Anaesth. [Evaluation Studies Research Support, Non-U.S. Gov't]. 2012;108:768-75.

40. Gobierno de Chile. Ministerio de Salud de Chile. Departamento de Epidemiología. Encuesta Nacional de Salud 2009-2010. Disponible en: http://epi.minsal.cl/estudiosy-encuestas-poblacionales/encuestas-poblacionales/ encuesta-nacional-de-salud/resultados-ens/.

41. Litman RS, Joshi GP. Malignant hyperthermia in the ambulatory surgery center: how should we prepare? Anesthesiology. [Editorial]. 2014;120:1306-8. 